\title{
Quasi-Dynamic Testing of an Optical Sensor for Measurements of the Blood Chamber Volume in the POLVAD Prosthesis
}

\author{
G. KOnIECZnY*, T. PUSTELny AND P. MARCZYŃSKi \\ Department of Optoelectronics, Silesian University of Technology, Akademicka 2A, 44-100 Gliwice, Poland
}

\begin{abstract}
The article presents further developments of an optical system to be used in temporary blood volume measurements in the Polish ventricular assist device. The research focuses on quasi-dynamic measurements (flow rate of $5 \mathrm{ml} / \mathrm{s}$ ). Characteristics of signals from selected configurations of optical transmitters and receivers are shown. A volume approximation using a PCA algorithm supported by a LRS function is introduced. Plans of further development are stated.
\end{abstract}

DOI: $10.12693 /$ APhysPolA.124.483

PACS: 42.79.-e, 42.81.-i, 07.07.Df, 47.63.Cb

\section{Introduction}

Heart diseases have become an alarming problem in the human population; therefore some methods of curing patients suffering from illnesses of the cardiovascular system must be found. Patients suffering from heart diseases can be divided into groups depending on the stage of the heart failure. According to the New York Heart Association (NYHA), there are four classes of the heart failure process. In mild cases of the heart failure, the use of drugs may be sufficient. The situation is different in the case of patients with end stage heart failures (class IV). Two decades ago, the only lifesaving option for those patients was heart transplant surgery. Nowadays, the heart support using additional blood pumping devices is practiced in a large group of patients. Ventricular assist devices (VAD) are used for reducing the load of the heart muscle, allowing a recovery of the human heart.

In Poland, the Polish ventricular assist device (POLVAD) is used in patients with an end-stage heart failure. The "Polish Artificial Heart Program" has been founded in order to improve the existing prosthesis solution, and to equip it with monitoring equipment, allowing an automation of the heart support process [1-8].

The POLVAD prosthesis (POLVAD-MEV model) has been used in Polish patients for over a decade now. The heart support process was automatized to the point of driving the prosthesis with fixed parameters (driving air pressure and pumping speed). Due to the lack of monitoring devices, an adjustment of the heart support process to the needs of the patient could not be achieved. In pulsatile, pneumatic prostheses, the temporary volume of blood filling the prostheses should be determined. This would allow to describe the state of the prosthesis, and

*corresponding author; e-mail: grzegorz.konieczny@polsl.pl to estimate the amount of blood pumped per minute cardiac output (CO).

Currently in the world the $\mathrm{CO}$ is measured applying VAD's blood-flow measurements, but the estimation of blood requires periodically controlled full-filling/full-ejection cycles. It does not provide reliable information about the amount of blood inside the prosthesis. Moreover, it requires very expensive noninvasive, ultrasound flow rate meters. Their construction cannot be miniaturized at the current state of art in electronics and ultrasonic transducers. Other approaches to this topic, permitting for dynamic temporary blood volume measurements, that might be incorporated in the construction of the prosthesis, proved to be not successful. One of the solutions allowing noninvasive blood chamber volume measurements in the POLVAD prosthesis was an acoustic system, basing on Helmholtz's resonator [9-11]. This method provided results with $10 \%$ uncertainty, and could be integrated with the prosthesis, but the need of an additional sensor chamber would hinder the use of the acoustic sensor in an implantable solution.

The novel solution, the optical measurement system, has been proposed [12]. The following paragraphs describe the advance in the development of the optical method.

\section{Quasi-dynamic investigations of the optical volume measurement system}

The optical system for measurements of the blood chamber volume of the POLVAD-EXT prosthesis has been evaluated by static tests at the Department of Optoelectronics. The obtained results indicate that the sensor can provide accurate results, allowing to assess the blood chamber volume with measurement uncertainties of $<10 \%$ of the volume range. It has been decided to extend the testing of the method using slow continuous flow rates of liquid pumping $(5 \mathrm{ml} / \mathrm{s})$. The article presents a methodology and the results of volume measurements under conditions of low dynamic flow rates. 
Compared with previous researches [12] the measurement stand and acquisition algorithm were modified. The modifications of the algorithm aimed at increase of the speed of the acquisition of data. The acquisition time of data from all the 384 tested configurations of LEDs and photodiodes was reduced from $1 \mathrm{~s}$ to $100 \mathrm{~ms}$.

The modifications of the measurement stand aimed at increasing the rate of water pumping. An additional module was constructed allowing to drive continuously a peristaltic pump. Additionally, the calibration of " $0 \mathrm{ml}$ " level, conducted each pumping cycle during static tests, proved obsolete in the new setup. The simple solution allowing for changing of the liquid pumping direction was developed (Fig. 1).

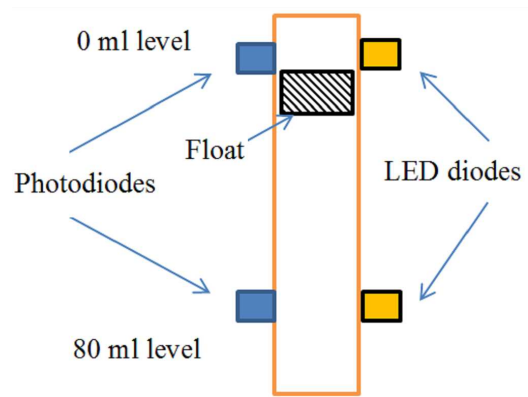

Fig. 1. Modified setup allowing for the change of liquid pumping direction.

The new approach guaranteed the change of liquid pumping direction at exact knowing blood chamber volumes $(0 \mathrm{ml}$ and $80 \mathrm{ml})$; therefore the calibration process was no longer necessary.

\section{Analysis of the results}

The optical system was tested at the Department of Optoelectronics. 40 series of full-filling/full-ejecting were performed. Due to the dynamic type of analysis, the time characteristics of voltages for different configurations of LEDs and photodiodes were obtained. An exemplary time characteristics is presented in Fig. 2.

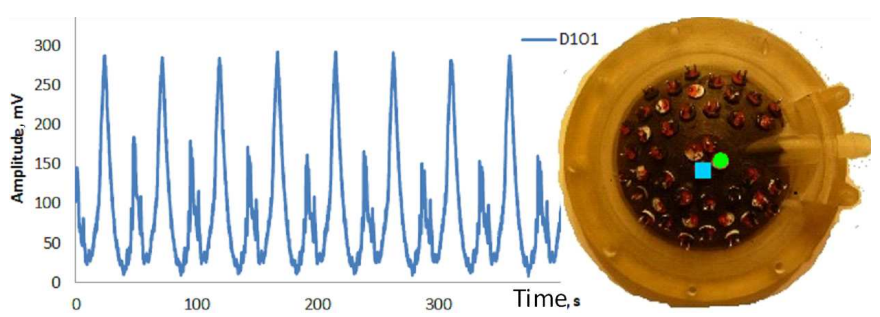

Fig. 2. Time-amplitude characteristics for configuration: diode 1-photodiode 1.

The characteristics shown in Fig. 2 is representative for a group of configurations revealing a recurrent nature. Since the data acquisition was made at a stable flow rate of liquid, the amplitude-volume graph could be constructed (Fig. 3).

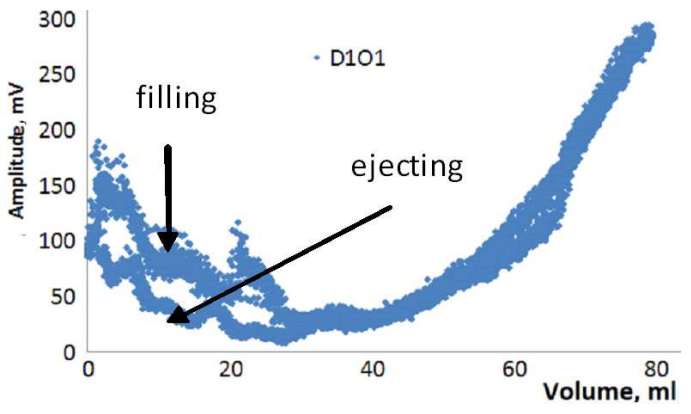

Fig. 3. Forty time-amplitude characteristics for configuration: diode 1-photodiode 1 .

It can be seen that the characteristic is adequate in the case of higher blood chamber volumes $(>40 \mathrm{ml})$. In the case of lower volumes it varies between filling and ejecting parts of each cycle, remaining repeatable within those parts in different cycles.

The results were used to analyse the PCA algorithm supported by the LRS feature selection function, with using a volume recognition algorithm [13]. A half of the results was used for learning purposes, the other half for testing the algorithms.

The recognition algorithm allows to recognize volumes of $0-80 \mathrm{ml}$ in $5 \mathrm{ml}$ steps. The objective function selected 86 out of 384 features (light emitter-receiver pairs). The results of volume assessment are shown in Fig. 4. The continuous line shows the actual volume of the blood chamber of the prosthesis. The step-like graph is constructed making use of the volume recognition algorithm.

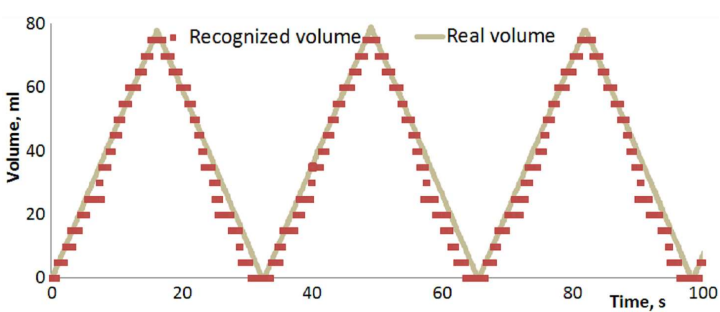

Fig. 4. Time-volume characteristics of a real and recognized blood chamber volume using 86 features.

It can be seen that the recognized volume plot follows the actual volume plot. The differences are within the $10 \%$ uncertainty range. The results of recognition were promising, but the number of features used by the algorithm was too large to use this method in the final prosthesis model.

This leads to modifications of the objective function the combinations allow to determine the blood chamber volume within the required error margin, making use of a lower number of features. The results of volume recognition concerning the same data set are shown in Fig. 5. 


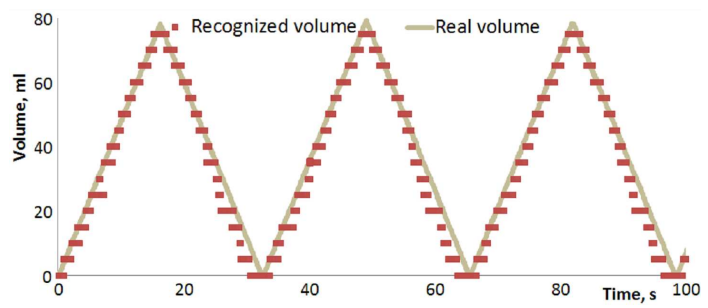

Fig. 5. Time-volume characteristics of a real and recognized blood chamber volume using 34 features.

Although the number of features was reduced by more than a half, the recognition plot still follows the shape of the real volume plot. The accuracy of recognition decreased in the case of volumes $<40 \mathrm{ml}$; but it was still acceptable. A further decrease of the number of features using the new objective function resulted in significant losses in volume assessment accuracy for middle volume range.

\section{Conclusions}

The modifications of the optical system allowed quasi-dynamic measurements. The measurement setup was also modified to allow a continuous change of the blood chamber volume of the prosthesis. The data acquisition speed was further improved, allowing for $100 \mathrm{~ms}$ acquisition time of measurement results from all 384 configurations. During 40 series of full-filling/full-ejecting, like in previous researches of the optical system, some configurations provide repeatable amplitude-volume characteristics. It allowed to improve the volume recognition algorithm aiming at improving the accuracy of assessment of the blood chamber volume, as well as limiting the number of required features (light emitter-receiver pairs).

The old objective function requires 86 features. The new one could work successfully with only 34 features. The number of optoelectronic elements used is still too large; therefore additional researches will have to be conducted in order to limit it still more.

Although the measurements were not static any more, the pumping conditions were still stable compared to those occurring in a heart support process. A verification will be required to determine whether the prosthesis will behave in a similar manner in actual dynamic conditions.

The volume estimation by means of the volume recognition algorithm allowed to estimate the blood chamber volume with $10 \%$ uncertainty. The method requires additional improvements regarding the number of features needed for a more accurate volume estimation.

Thanks to quasi-dynamic researches the new optical method will be prepared to dynamic measurements conducted at the Foundation of Cardiac Surgery Development, Zabrze, Poland.

\section{Acknowledgments}

The investigations were partially financed within the Polish National Centre of Science NCN (Cracow, Poland), within the grant No. 4896/B/TO2/2011/40.

M.Sc. Grzegorz Konieczny is a beneficiary of the "SWIFT (Scholarships Supporting Innovative Technology Forum)" project POKL.08.02.01-24-005/10 co-financed by the European Union within the European Social Fund.

\section{References}

[1] J. Pacholewicz, M. Zakliczyński, A. BarańskaKosakowska, G. Religa, P. Siondalski, E. Kucewicz, P. Nadziakiewicz, M. Zembala, Heart Lung Transplant. 28, S98 (2009).

[2] A. Milenin, M. Kopernik, D. Jurkojc, M. Gawlikowski, T. Rusin, M. Darłak, R. Kustosz, Acta Bioeng. Biomech. 14, 49 (2012).

[3] http://www.polskiesztuczneserce.pl/.

[4] A. Sobotnicki, T. Pałko, J. Mocha, M. Czerwiec, J. Med. Informat. Technol. 19, 117 (2012).

[5] K. Gut, Acta Phys. Pol. A 114, A121 (2008).

[6] M. Gawlikowski, D. Komorowski, Adv. Soft Comput. 45, 558 (2007).

[7] M. Gawlikowski, T. Pustelny, R. Kustosz, J. Phys. IV: JP (France) 137, 73 (2006).

[8] K. Barczak, T. Pustelny, D. Dorosz, J. Dorosz, Europ. Phys. J. Spec. Top. 154, 11 (2008).

[9] G. Konieczny, Z. Opilski, T. Pustelny, Acta Phys. Pol. A 120, 688 (2011).

[10] Z. Opilski, G. Konieczny, T. Pustelny, A. Gacek, R. Kustosz, Bull. Pol. Acad. Sci., Techn. Sci. 59, 429 (2011).

[11] G. Konieczny, Z. Opilski, T. Pustelny, M. Gawlikowski, BioMed. Eng. OnLine 11, 72 (2012).

[12] G. Konieczny, T. Pustelny, Acta Phys. Pol. A 122, 962 (2012).

[13] P. Marczyński, A. Szpakowski, C. Tyszkiewicz, T. Pustelny, Acta Phys. Pol. A 122, 847 (2012). 